\title{
Erratum to: Dielectric function of graphene based quantum dot under the uniaxial stress
}

\author{
A. Phirouznia ${ }^{1, a}$, N. Akbari ${ }^{2}$, S. Lotfi ${ }^{2}$, and K. Jamshidi-Ghaleh ${ }^{1}$ \\ 1 Department of Physics, Azarbaijan University of Tarbiat Moallem, 53714-161 Tabriz, Iran \\ 2 Department of Laser and Optical Engineering University of Bonab, 5551761167 Bonab, Iran
}

Eur. Phys. J. B 85: 259 (2012)

Received 4 August 2012

Published online 10 September 2012 - (C) EDP Sciences, Società Italiana di Fisica, Springer-Verlag 2012

We realized that due to an unfortunate mistake the expression of the basis vectors has been omitted in the second page of the paper. When the stress is applied along the $x$ direction, the basis vectors, generating the real space points can be written as:

$$
\mathbf{a}_{1}=\frac{a}{2}(3 \eta, \sqrt{3}), \quad \mathbf{a}_{2}=\frac{a}{2}(3 \eta,-\sqrt{3})
$$

$\eta$ is the parameter characterizing the stress process.

In addition both parts of Figure 2 presenting the imaginary part of the $\epsilon_{x x}$. Consequently the vertical axis of the inner part of this figure should be $\operatorname{Im}\left(\epsilon_{x x}\right)$. We apologize for the inconvenience that these mistakes may have caused.

\footnotetext{
${ }^{a}$ e-mail: Arash.phi@gmail.com
} 